PRESENTACIÓN

\title{
Corrupción y diseños institucionales en América Latina
}

\author{
CARLOS ALZA BARCO* \\ Director, Escuela de Gobierno y Políticas Públicas PUCP \\ carlos.alza@pucp.edu.pe \\ DIEGO A. SALAZAR MORALES** \\ Docente de la Escuela de Gobierno y Políticas Públicas PUCP \\ d.salazar@phd.hertie-school.org
}

https://doi.org/10.18800/rcpg.201702.000

¿Qué es la corrupción?, ¿cómo detectarla? y ¿cómo combatirla? Son preguntas que emergieron en los últimos años en América Latina en un contexto marcado por su posicionamiento en la palestra mundial como una de las regiones más corruptas del planeta. Hacia el año 2013, las denuncias periodísticas por corrupción involucraron a cerca de trece países en la región (Gestión, 2017). Esta situación dio por sentada que la corrupción era (es) el principal tópico de preocupación en la región. Según Transparencia Internacional (2017), en promedio, América Latina se ubica en el cuartil superior entre los países corruptos a nivel mundial, situación preocupante para la región. Los altos índices de corrupción no sólo afectan la calidad y cantidad de gasto público, encarecen la inversión privada, reducen el acceso a los servicios públicos para la ciudadanía y detienen el desarrollo, sino que sus efectos también son sistémicos: influyen en las relaciones cotidianas entre ciudadanía y Estado y las distorsiona creando una institucionalidad paralela. En otras palabras, genera una cultura de transgresión.

A pesar de que la corrupción está presente en la agenda pública latinoamericana desde hace décadas, los estudios académicos no se han preocupado por identificar los mecanismos o estrategias que sirvan de insumo para el diseño de políticas públicas. En cambio, desde 1990, las investigaciones desarrolladas por autores como Geddes y Ribeiro (1992), Weyland (1998), así como Della Porta

\footnotetext{
* Director de la Escuela de Gobierno y Políticas Públicas de la PUCP. Magíster en Regulación por la London School of Economics and Political Science en el Reino Unido.

** MSc. en Políticas Públicas y Administración por la London School of Economics and Political Science. Licenciado en Ciencia Política por la Universidad Nacional Mayor de San Marcos.
} 
y Vanuci (1999), se preocuparon por la relación entre corrupción y democracia como una relación de dependencia mutua. Esto, según los autores, se debe a que los procesos de democratización en la región habrían incrementado la cantidad de actores demandando accesos ilegales al Estado. Más adelante, hacia el ańo 2000, autores como Davis, Camp y Coleman (2004), Seligson (2006) y Canache y Allison (2005) se enfocaron en el rol de las percepciones de la corrupción en la ciudadanía, su medición y, específicamente, sobre la percepción de este fenómeno y su impacto en la confianza de las instituciones. Pocos académicos, en cambio, pusieron énfasis en explorar la relación con los procesos de liberalización ocurridos durante los años noventa (Whitehead, 2000), así como analizar la corrupción en procedimientos estatales y en procesos de negociación en concesiones públicas (Guash y Straub, 2009).

Esta inquietud sobre la corrupción estuvo, y está, marcada por la preocupación entre corrupción y democracia, así como una preponderancia de los estudios sobre la "percepción de la corrupción». En los hechos, a través de un análisis desarrollado sobre las publicaciones arbitradas en materia de corrupción entre el periodo 2007 y 2010, encontramos que existen 166 publicaciones que tienen como centro de su investigación a la corrupción en América Latina. Entre los tópicos más comunes se encuentran aquellas que exploran los efectos de la percepción de la corrupción en la democracia (11), en la dinámica nacional y regional (7), en el contexto subnacional (6), su relación con la pobreza (4), con procesos de privatización (3) y escándalos públicos (3). Son menos comunes los estudios relacionados a género y corrupción, cultura popular e infraestructura.

La particular preocupación por las percepciones de la corrupción tiene su origen en la disponibilidad de datos sobre este fenómeno. Organismos internacionales como Transparencia Internacional, GIZ, UNDOC, IACA, el Latinobarómetro, han promovido estudios sobre la corrupción basados en mediciones de las percepciones del fenómeno. Algunas de estas entidades innovaron en la forma de analizar la tasa de victimización que enfrentan los ciudadanos. Gran parte de estas mediciones fomentaron investigaciones sobre las percepciones de la población y su relación con la confianza institucional, la permanencia y apoyo a la democracia. Sin embargo, las miradas novedosas que apuntan a identificar mecanismos institucionales que generan la corrupción, con el objetivo de formular políticas públicas, no han sido frecuentes. Ciertamente, esta necesidad está vinculada a limitaciones propias del estudio del fenómeno de la corrupción. Tal como Landé (1983) hiciera en los años ochenta para los estudios sobre el clientelismo y patrimonialismo, los estudios de la corrupción aún presentan limitaciones en: (i) la comprensión plena de los límites de lo 
que es o no corrupto, (ii) en la posibilidad de observar el fenómeno como una interacción cotidiana y, consecuentemente, (iii) en su mensurabilidad. Precisamente, esclarecer estas limitaciones permitiría un mejor entendimiento de las políticas públicas anticorrupción, toda vez que — tal como Hood (2007) mencionó- en la gestión pública el mundo de los objetivos y las mediciones comanda los objetivos de política pública. En otras palabras, lo que se mide, vale decir, lo que se visibiliza, es lo que se implementa.

En tal sentido, la limitación por comprender la corrupción más allá de los escándalos políticos, como problemas de percepción o en su relación con la democracia, ha opacado la mirada sobre sus causas. La corrupción ha sido vista por mucho tiempo como una variable independiente. La ciencia política ha sido un importante exponente de este particular enfoque, pero esto no es suficiente. Urge una mirada desde la administración pública que se preocupe por los diseños institucionales que sirven como fuentes de la corrupción, que identifique los instrumentos de política pública que han servido para combatir este fenómeno y, finalmente, que analice el rol de las instituciones que han sido creadas para tal fin. Urge, en otras palabras, una mirada instrumental sobre las políticas anticorrupción.

Esta es una tarea que se alcanzará en el largo plazo y con el esfuerzo articulado de agencias de cooperación internacional, Estado y academia. También es una tarea a desarrollarse en tres etapas: una primera que promueva una compresión profunda de la corrupción, conforme al contexto institucional y cultural de nuestros países. Esto implica la identificación de definiciones, mecanismos y prácticas comunes de corrupción en los países de América Latina a través de investigaciones que se enfoquen en los mecanismos causales de la corrupción, en especial en los servicios públicos. Una segunda etapa se enmarca en el análisis de los diseños institucionales de las políticas públicas y cómo estos favorecen — generan de forma intencionada o no- la corrupción. Este es un paso importante, ya que posicionaría los estudios sobre la corrupción en la región a la par de nuevas aproximaciones a nivel internacional (Manion, 2004; Ferraz de Almeida, 2015). Finalmente, una tercera etapa se concentraría en evaluar las intervenciones estatales en materia de lucha contra la corrupción identificando qué intervenciones han sido efectivas y cuáles no.

Transitar de la primera a la tercera etapa es un camino largo y dificultoso. Precisamente, este número especial de la Revista de Ciencia Política y Gobierno de la PUCP se enmarca en las dos primeras etapas: comprender los mecanismos y prácticas de la corrupción en países de América Latina y analizar los diseños institucionales que promueven o sostienen la corrupción. Consideramos que la tercera etapa (evaluación de las políticas anticorrupción) merece una atención especial y 
—a pesar de los esfuerzos desplegados en este número especializado- requiere de un nuevo volumen, especialmente diseñado para evaluar las políticas públicas desarrolladas en nuestros países en cuanto al combate contra la corrupción.

En este número presentamos investigaciones que apuntan a la comprensión del fenómeno de la corrupción. Crespo, desde Ecuador, analiza la relación entre corrupción y la confianza institucional. El autor verifica la tesis de Rothstein (2000) sobre la relación entre ambas variables y cómo estas establecen un círculo vicioso que solo podría ser modificado con grandes reformas institucionales. Ya hacia 2011, Rothstein menciona que este tipo de reformas solo se podrían desarrollar a gran escala, el autor las denomina reformas «big bang». Crespo toma las principales propuestas de Rothstein y demuestra que tanto la confianza interpersonal como establecida entre ciudadanos y Estado es clave para la gestación de la corrupción. Esta es una relación que parece tautológica, sin embargo, tal como Rothstein y Teorell (2008) señalan, cuando el fenómeno alcanza niveles sistemáticos es difícil aislar variables sin que estas generen —y reciban- efectos de otras.

También en este número, Ramos, Oblitas y Zúñiga hacen un recuento de los métodos de estudio de la corrupción. El artículo describe investigaciones desarrolladas desde métodos y análisis tradicionales de la corrupción —al cual los autores denominan observacionales — hasta los diseńos experimentales. Los autores listan los principales diseños experimentales en el análisis y estudio de la corrupción: (i) los experimentos de laboratorio, (ii) los experimentos de campo y (iii) los diseños experimentales de encuestas. Ambos artículos, tanto el de Crespo y el de Ramos et al., apuntan a la comprensión de los aspectos definicionales y metodológicos de la corrupción.

El segundo grupo de artículos presentes en este número de esta Revista posiciona a la corrupción como variable dependiente y analiza los factores que la generan y promueven. En este grupo se encuentra el trabajo de Gazenov, quien, a través de análisis de regresión multivariante sobre las principales bases de datos internacionales en la materia, analiza las principales teorías que hay detrás de la explicación de los actos corruptos. El autor encuentra que variables tales como el imperio de ley (rule of law), violencia, estabilidad relativa de un país y la riqueza monetaria tienen una correlación significativa con la presencia de actos corruptos. En este bloque también se encuentra el análisis desarrollado por Pozsgai, quien analiza las causas que generan la permanencia del statu quo en cuanto a la oferta de políticas públicas de lucha contra la corrupción. Pozsgai menciona que la voluntad política detrás del recambio en las políticas de lucha contra la corrupción podría explicarse a partir de elementos como la (i) ineficiencia en procesos de entrega oportuna de servicios públicos que genera la corrupción, (ii) la percepción que los ciudadanos tienen sobre la comisión de 
actos corruptos de parte de sus élites gobernantes, (iii) la intolerancia adquirida de la corrupción a partir de la aparición de movimientos internacionales que luchan contra este fenómeno y (iv) la negligencia que se acumularía en el sistema político por falta de presión ciudadana para el recambio de rumbo en la implementación de políticas públicas anticorrupción. El autor presenta su análisis recurriendo a casos mediáticos de corrupción ocurridos en las últimas décadas.

Finalmente, y en este segundo bloque de artículos se encuentran los estudios empíricos de Chiroque sobre el combate a la corrupción en la Superintendencia Nacional de Administración Tributaria del Perú (Sunat), así como el estudio de Melgar sobre el establecimiento de una red de corrupción en el Gobierno Regional de Áncash. El primero presenta un análisis detallado sobre cómo, a partir de escándalos de corrupción en la Sunat, se creó la Oficina de Fortalecimiento Ético y Lucha contra la Corrupción. Esta oficina tuvo severas limitaciones para la implementación de una política anticorrupción efectiva dentro de la entidad. Entre los factores que limitaron el éxito de la política anticorrupción el autor menciona: (i) la excesiva influencia política de la alta dirección durante la investigación de casos de corrupción, (ii) la presencia de vacíos regulatorios y legales en el funcionamiento del Órgano Interno de Control de la Sunat, (iii) el ineficiente sistema de identificación de riesgos de corrupción, (iv) las limitadas capacidades técnicas entre las áreas encargadas del control y (v) los limitados recursos logísticos y presupuestales. El documento de Melgar explica los factores que contribuyeron al establecimiento de una de las redes de corrupción más sorprendentes de la última década en el Perú. A través de 67 entrevistas a actores clave en las provincias involucradas en actos de corrupción, revisión de documentos y notas periodísticas, la autora muestra cómo se estableció la red de César Álvarez, llamado la Bestia. Melgar descubre tres «mecanismos causales» que favorecieron que dicho gobernador llegara a extender sus redes a través de todo el departamento: (i) la administración de ingentes recursos económicos provenientes del canon, (ii) las alianzas conformadas a nivel local y nacional, y (iii) los vínculos del gobernador con el hampa. Tales factores abrieron la posibilidad de construir redes capaces de neutralizar la acción de opositores mediante amenazas creíbles a su integridad física. El estudio de Melgar es clave para la compresión de la corrupción subnacional y creemos que estructura un ejercicio a ser replicable en futuras investigaciones.

Con este número, la Escuela de Gobierno y los editores buscan contribuir al debate sobre el estudio de la corrupción en América Latina. Es importante reconocer, empero, que el camino es aún largo y que este es un primer paso en la tarea de combatir la corrupción como el mal endémico que limita el desarrollo de los países de la región. 


\section{REFERENCIAS}

Canache, D. \& Allison, M. E. (2005). Perceptions of political corruption in Latin American democracies. Latin American Politics and Society, 47(3), 91-111. https://doi.org/10.1353/ lap.2005.0031

Davis, C. L., Camp, R. A. \& Coleman, K. M. (2004). The influence of party systems on citizens' perceptions of corruption and electoral response in Latin America. Comparative Political Studies, 37(6), 677-703. https://doi.org/10.1177/0010414004265879

Della Porta, D. \& Vannucci, A. (1999). Corrupt exchanges: Actors, resources, and mechanisms of political corruption. Nueva York: Transaction Publishers.

Ferraz de Almeida, M. (2015). Corruption by design and the management of infrastructure in Brazil: Reflections on the Programa de Aceleracao do Crescimento - PAC.

Geddes, B. \& Ribeiro, A. (1992). Institutional Sources of Corruption in Brazil. Third World Quarterly, 13(4), 641-661. https://doi.org/10.1080/01436599208420302

Gestión (2017). Caso Lava Jato: Cronología de las investigaciones por corrupción en Brasil. Recuperado el 16 de octubre de 2017 de https:/gestion.pe/peru/politica/lava-jato-cronologiainvestigaciones-corrupcion-brasil-113656

Guasch, J. L. \& Straub, S. (2009). Corruption and concession renegotiations: Evidence from the water and transport sectors in Latin America. Utilities Policy, 17(2), 185-190. https://doi. org/10.1016/j.jup.2008.07.003

Hood, C. (2007). Public service management by numbers: Why does it vary? Where has it come from? What are the gaps and the puzzles? Public Money and Management, 27(2), 95-102. https://doi.org/10.1111/j.1467-9302.2007.00564.x

Landé, C. H. (1983). Political clientelism in political studies: Retrospect and prospects. International Political Science Review, 4(4), 435-454. https://doi.org/10.1177/019251218300400403

Manion, M. (2004). Corruption by design: Building clean government in mainland China and Hong Kong. Cambridge: Harvard University Press.

Rothstein, B. (2000). Trust, social dilemmas and collective memories. Journal of Theoretical Politics, 12(4), 477-501. https://doi.org/10.1177/0951692800012004007

Rothstein, B. (2011). Anti-corruption: the indirect 'big bang'approach. Review of International Political Economy, 18(2), 228-250. https://doi.org/10.1080/09692291003607834

Rothstein, B. \& Teorell, J. (2008). What is quality of government? A theory of impartial government institutions. Governance, 21(2), 165-190. https://doi.org/10.1111/j.1468-0491.2008.00391.x

Seligson, M. A. (2006). The measurement and impact of corruption victimization: Survey evidence from Latin America. World Development, 34(2), 381-404. https://doi.org/10.1016/j. worlddev.2005.03.012

Transparencia Internacional (2017). Índice de Percepción de la Corrupción 2016: Urge abordar círculo vicioso de corrupción y desigualdad. [online] www.transparency.org. https://www.transparency.org/news/pressrelease/indice_de_percepcion_de_la_corrupcion_ 2016_urge_abordar_circulo_vicioso_de

Weyland, K. G. (1998). The politics of corruption in Latin America. Journal of Democracy, 9(2), 108-121. https://doi.org/10.1353/jod.1998.0034

Whitehead, L. (2000). High-level political corruption in Latin America: a transitional phenomenon. En J. S. Tulchin y R. H. Spach (eds.), Combating corruption in Latin America (pp. 107-129). Washington, DC: Woodrow Wilson Center Press. 